\title{
Reorientation of magnetic anisotropy in epitaxial cobalt ferrite thin films
}

\author{
A. Lisfi,,${ }^{1} *$ C. M. Williams, ${ }^{1}$ L. T. Nguyen, ${ }^{2}$ J. C. Lodder,${ }^{2}$ A. Coleman, ${ }^{1}$ H. Corcoran, ${ }^{1}$ A. Johnson, ${ }^{1}$ P. Chang, ${ }^{1}$ \\ A. Kumar, ${ }^{1}$ and W. Morgan ${ }^{1}$ \\ ${ }^{1}$ Department of Physics, Morgan State University, 1700 E. Cold Spring Lane, Baltimore, Maryland 21251, USA \\ ${ }^{2}$ SMI, MESA + Institute for Nanotechnology, University of Twente, P.O. Box 217, 7500 AE Enschede, The Netherlands
}

(Received 27 March 2007; revised manuscript received 23 May 2007; published 3 August 2007)

\begin{abstract}
Spin reorientation has been observed in $\mathrm{CoFe}_{2} \mathrm{O}_{4}$ thin single crystalline films epitaxially grown on (100) $\mathrm{MgO}$ substrate upon varying the film thickness. The critical thickness for such a spin-reorientation transition was estimated to be $300 \mathrm{~nm}$. The reorientation is driven by a structural transition in the film from a tetragonal to cubic symmetry. At low thickness, the in-plane tensile stress induces a tetragonal distortion of the lattice that generates a perpendicular anisotropy, large enough to overcome the shape anisotropy and to stabilize the magnetization easy axis out of plane. However, in thicker films, the lattice relaxation toward the cubic structure of the bulk allows the shape anisotropy to force the magnetization to be in plane aligned.
\end{abstract}

DOI: 10.1103/PhysRevB.76.054405

PACS number(s): 75.70.Ak, 75.30.Gw

The importance of magnetic anisotropy is well recognized in many technical applications such as magnetic and magneto-optic recording. The large interest for high anisotropies is motivated by technological demands such as increasing the magnetic recording density. With large anisotropy, the superparamagnetic limit can be pushed down, and a stable magnetization can be promoted in ultrasmall nanosized magnetic structures, which are needed in advanced media for ultrahigh density recording. Besides the intrinsic anisotropy of the bulk, other sources of anisotropy may be enhanced in artificial structures and contribute to their magnetic properties. Depending on their relative orientations and magnitudes, the involved anisotropies may compete between each other, leading to spin-reorientation phenomena in the system. For example, the broken symmetry at the interfaces in ultrathin films generates a perpendicular anisotropy, which overcomes the shape anisotropy. ${ }^{1}$ However, increasing the layer thickness reduces the ratio between the surface and the volume atoms, leading to an in-plane alignment of the easy axis. $^{2}$ In obliquely sputtered metallic thin films, we established the existence of an in-plane reorientation of magnetic anisotropy. ${ }^{3}$ Depending on the film thickness and due to the shadow effect during the growth, the layer can develop columns or nuclei able to confine the anisotropy parallel or perpendicular to the longitudinal direction (projection of the incident beam in the film plane).

Ferrites cover a large family of oxides, including soft as well as hard magnetic materials. Hard ferrites such as the hexagonal $\left(\mathrm{BaFe}_{12} \mathrm{O}_{19}\right)$ and the spinel $\left(\mathrm{CoFe}_{2} \mathrm{O}_{4}\right)$ are particularly attractive for magnetic and magneto-optic recording applications due to their large magnetocrystalline anisotropy and high chemical stability. Recent studies demonstrated that integrating cobalt ferrite as a pinning layer in the spin valve architecture can strongly enhance the magnetoresistance effect of the sandwiched structure. ${ }^{4}$ In epitaxial hexaferrite thin films, the uniaxial magnetocrystalline anisotropy is strong enough to dominate all the other sources of anisotropy and to keep the spin alignment constant regardless the film thickness and the preparation conditions. ${ }^{5}$ However, cobalt ferrite represents a unique case among all the ferrite families since its large magnetostriction can promote the strain anisotropy to prevail in low dimensional structures such as thin films.

In this paper, we first report on the thickness dependence spin reorientation in epitaxial $\mathrm{CoFe}_{2} \mathrm{O}_{4}$ thin films. After being confined to the normal to the film plane in thinner layers, the easy axis switches to become in plane aligned in thicker films. Our samples have been grown by pulsed laser deposition on (100) magnesium oxide substrate. $\mathrm{MgO}$ is expected to be a good template to achieve an epitaxial growth since its lattice parameter $(4.21 \AA)$ is quasiequal to the half of that of the bulk $\mathrm{CoFe}_{2} \mathrm{O}_{4}(8.38 \AA)$. Consequently, one film lattice may fit on four substrate lattice units since their mismatch is estimated to be as small as $0.48 \%$. More detail about the fabrication procedure is given in Ref. 5. A series of samples having thicknesses in the $50-500 \mathrm{~nm}$ range has been prepared by varying the number of laser shots during deposition. In this paper, we report about three film thicknesses, namely, 60, 240, and $400 \mathrm{~nm}$.

Figure 1 shows the in-plane and perpendicular magnetic loops of the three films $(60,240$, and $400 \mathrm{~nm}$ thick). The measurement has been performed using a vibrating sample magnetometer (VSM). In the thinner layer [Fig. 1(a)], the perpendicular loop exhibits a significant shearing induced by the demagnetizing field and reveals the existence of a large hysteresis with a coercivity of $0.6 \mathrm{~T}$. However, the behavior of the in-plane magnetization consists of two regimes. A fast jump with a very small hysteresis appears at low fields, whereas a linear increase is noticed at large fields without saturation even at $3 \mathrm{~T}$. The huge difference between the inplane and out-of-plane loops suggests that the magnetization easy axis is aligned perpendicular to the film plane. It is important to point out that the behavior of the in-plane magnetization deviates from that predicted by the StonerWohlfarth model for an oriented uniaxial anisotropy. ${ }^{6}$ In such a model, the magnetization mechanism is purely reversible when the direction of the applied field is parallel to the hard axis. However, the vertical switching with hysteresis in the in-plane magnetization [Fig. 1(a)] reveals the existence of an additional component of anisotropy, oriented parallel to the film plane. Upon increasing the film thickness [Fig. 1(b)], the coercivity of the perpendicular loop $H_{c \perp}$ is considerably reduced, whereas the one of the in-plane magnetization $H_{c \|}$ 


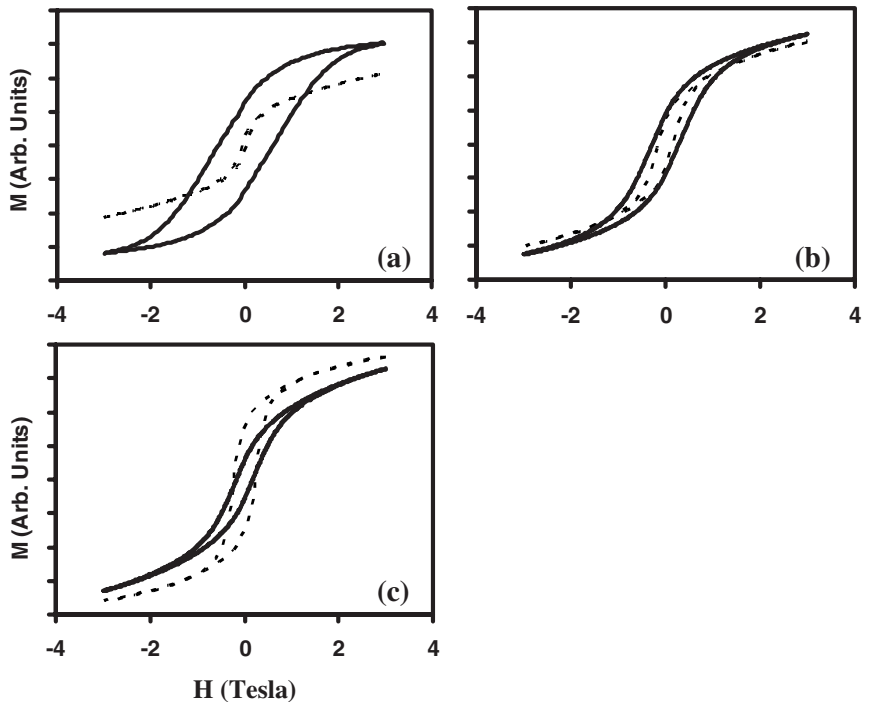

FIG. 1. In-plane (dashed line) and perpendicular (solid line) hysteresis loops of (a) $60 \mathrm{~nm}$, (b) $240 \mathrm{~nm}$, and (c) $400 \mathrm{~nm} \mathrm{CoFe}{ }_{2} \mathrm{O}_{4}$ thick films. While the easy axis is aligned out of plane at small thickness, the film plane becomes the preferential direction for the magnetization at large thickness.

shows a substantial increase. Moreover, the intermediate thickness represented by Fig. 1(b) shows two major effects in the magnetization behavior. (a) The difference between the in-plane and out-of-plane hystereses becomes insignificant. (b) At large field, both loops do not saturate but show a linear increase with a similar slope in both directions. Such a result suggests that our intermediate thickness $(240 \mathrm{~nm})$ is approaching the critical thickness $t_{c}$ considered as the crossover for the spin-reorientation transition. At $t_{c}$, both directions (in plane and perpendicular) are expected to be magnetically equivalent. A further increase of the film thickness leads to a drastic change in the magnetic properties, as illustrated by Fig. 1(c). The vertical switching of the magnetization and the large hysteresis of the in-plane loop confirm that the film plane becomes the preferential direction of the magnetization. Figure 1 shows that the orientation of the easy axis is controlled by the film thickness and illustrates clear evidence of the spin-reorientation transition. Several effects may be responsible for the hysteretic behavior of both loops (inplane and perpendicular) and can be listed as follows: (a) possible involvement of a cubic anisotropy can induce a hysteresis in both directions (in plane and out of plane). The cubic anisotropy can be inherited from the cubic symmetry of the bulk $\mathrm{CoFe}_{2} \mathrm{O}_{4}$ if the film lattice is undistorted. With cubic anisotropy, the magnetization will be always forced to be in plane aligned due to the shape anisotropy as is the case for the thicker film [Fig. 1(c)]. However, the out-of-plane alignment of the easy axis in the thinner layer [Fig. 1(a)] can hardly be explained by the cubic symmetry. (b) In the case of uniaxial anisotropy, a perpendicular spin alignment similar to that illustrated by Fig. 1(a) can be stabilized. However, the hysteretic behavior of the magnetic loops in both directions (in plane and perpendicular) can be seen in the following cases: (i) nonoriented films with a polycrystalline structure and random distribution of the easy axes and (ii) epitaxial

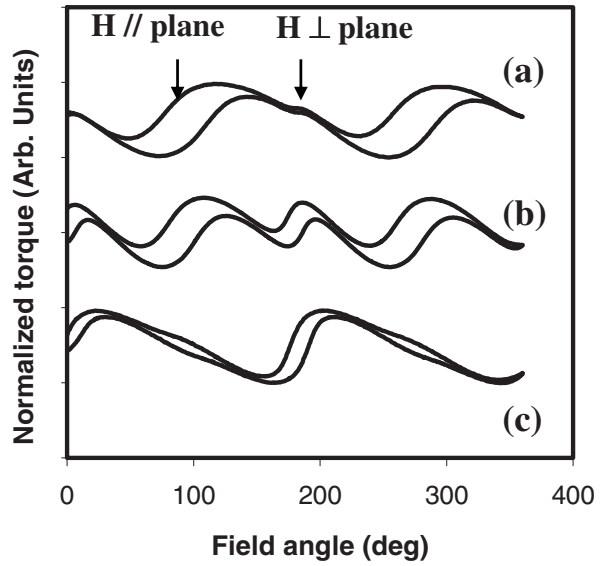

FIG. 2. Out-of-plane torque curves measured at $1.7 \mathrm{~T}$ for (a) $60 \mathrm{~nm}$, (b) $240 \mathrm{~nm}$, and (c) $400 \mathrm{~nm} \mathrm{CoFe} \mathrm{O}_{4}$ thick films. The graphs show two competing uniaxial anisotropies. At small thickness, the perpendicular anisotropy dominates, whereas at large thickness the in-plane component takes the lead.

layers where two anisotropies with different orientations (out of plane and in plane) can coexist. For that particular case, the two anisotropies must be acting or prevailing in separated areas of the layers. Otherwise, their superposition will lead to one effective anisotropy oriented parallel or perpendicular to the film plane without any hysteresis along the hard direction. In order to clarify all these points, we conducted additional investigations including anisotropy measurement performed by using torque magnetometer and structural analyses made by atomic force microscopy (AFM) and x-ray diffraction. Figure 2 depicts the out-of-plane torque curves of the 60,240 , and $400 \mathrm{~nm}$ thick films. In such measurements, a constant rotating field of $1.7 \mathrm{~T}$ is applied to the sample and the field orientation lies along the normal and parallel directions to the film plane at $0^{\circ}$ and $90^{\circ}$, respectively. In contrast to the cubic anisotropy of the bulk, the torque curve periodicity $\left(180^{\circ}\right)$ in the thinner layer [Fig. 2(a)] confirms the domination of oriented uniaxial anisotropy. Moreover, the existence of a large rotational hysteresis at $90^{\circ}$ reveals two important points. (a) The anisotropy field $H_{k}$ in such film is much higher than the external applied field during the measurement $(1.7 \mathrm{~T})$. (b) The easy axis is aligned perpendicular to the film plane since the reversible and irreversible (hysteresis) behaviors of the magnetization occur when the field direction approaches the easy and hard axes, respectively. It is important to point out the existence of an anomalous effect illustrated by a small kink in the torque curve. This kink exhibits a periodic behavior of $180^{\circ}$ and is localized at field angles close to the normal to the film plane $\left(0^{\circ}\right.$ and $\left.180^{\circ}\right)$. The kink could be attributed to a small in-plane uniaxial anisotropy responsible for the hysteretic vertical switching in the in-plane magnetization of Fig. 1(a). Increasing the film thickness induces drastic changes, which can be listed from the torque curve of Fig. 2(b) as follows: (a) a significant reduction of the rotational hysteresis of the out-of-plane uniaxial anisotropy and (b) the kink observed in the thinner layer, which is largely enhanced to be clearly identified as an in-plane anisotropy with a large rotational hysteresis and a magnitude approaching that of the perpendicular anisotropy. 


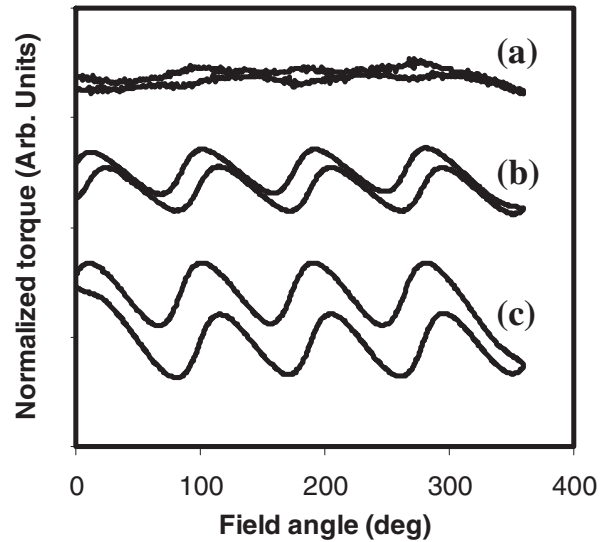

FIG. 3. In-plane torque curves measured at $1.7 \mathrm{~T}$ for (a) $60 \mathrm{~nm}$, (b) $240 \mathrm{~nm}$, and (c) $400 \mathrm{~nm} \mathrm{CoFe}_{2} \mathrm{O}_{4}$ thick films. While the inplane magnetization is quasi-isotropic at small thickness, a cubic anisotropy is progressively developed upon increasing the film thickness.

Further increase of the film thickness leads to a surprising result, as illustrated by Fig. 2(c). Despite a persistence of rotational hysteresis around $90^{\circ}$, the out-of-plane anisotropy vanishes, whereas the in-plane component arises and takes the lead. The out-of-plane torque curves confirm the coexistence of two competing uniaxial anisotropies. Moreover, the existence of two separated components of rotational hysteresis indicates a double switching of the magnetization, which can be realized only if both anisotropies (in plane and perpendicular) are prevailing in separated areas of the film. Figure 3 shows the in-plane torque curves of the three different samples $(60,240$, and $400 \mathrm{~nm}$ thick). In such a measurement, the rotating magnetic field remains parallel to the film plane and lies along the [010] and [001] crystallographic axes at $45^{\circ}$ and $135^{\circ}$, respectively. It can be clearly observed that the in-plane anisotropy exhibits a strong dependence on the film thickness. In the thinner layer [Fig. 3(a)], the flatness of the torque curve reveals the quasi-isotropic behavior of the magnetization in the film plane. However, upon increasing the film thickness, the in-plane anisotropy starts to develop, as illustrated by the substantial increase in magnitude and hysteresis of the torque curve. The periodicity $\left(90^{\circ}\right)$ of the torque curve is a good illustration of the existence of a cubic anisotropy in thicker films, where the easy axis lies along the [010] and [001] axes. The thickness dependence of both torque and magnetic loop in the prepared series of samples reveals two important results. (a) While a strong perpendicular uniaxial anisotropy controls the spin alignment in thinner films, a cubic anisotropy prevails in thicker films with an in-plane easy axis. (b) The critical thickness $t_{c}$ for the spin-reorientation transition was estimated to be around $300 \mathrm{~nm}$. The crucial question arises as to what are the sources of anisotropies involved in the control of the spin alignment in our layers. Generally, spin reorientation in thin films results from the competition between several uniaxial anisotropies such as (a) the surface anisotropy $K_{s}$, induced by the layer interfaces (film/substrate and film/vacuum), (b) the volume anisotropy $K_{v}$, which is generated by the spin-orbit coupling in both states (strained and relaxed) of the film

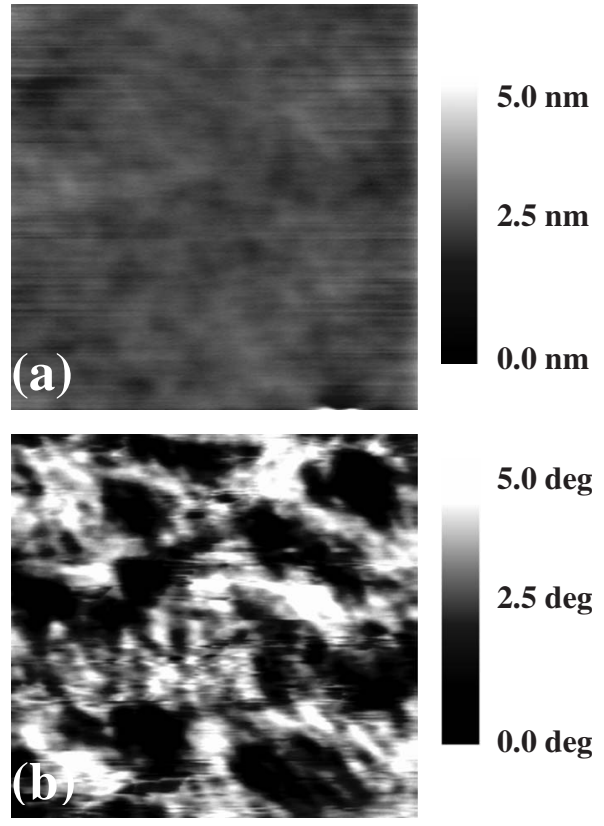

FIG. 4. $\left(3 \times 3 \mu \mathrm{m}^{2}\right)$ (a) AFM and (b) MFM images of as-grown $\mathrm{CoFe}_{2} \mathrm{O}_{4}$ film.

lattice, and (c) the shape anisotropy of the film, which represents the dipolar interactions and is proportional to the square of the film magnetization. The total uniaxial anisotropy energy $E_{a}$ in a thin film structure can be expressed as follows: $E_{a}=2 K_{s} / t+K_{v}-2 \pi M^{2}$, where $t$ and $M$ are the thickness and the magnetization of the film, respectively. A positive $E_{a}$ indicates a perpendicular alignment of the easy axis, whereas a negative $E_{a}$ describes an in-plane orientation of the magnetization. It is important to notice that the anisotropy $K_{s}$ due to the broken symmetry at the surface can be safely ignored in our films, due to their large thicknesses (more than $50 \mathrm{~nm}$ ). To estimate the shape anisotropy in our structures, we determined the film magnetization from the VSM measurement $\left(M=320 \mathrm{emu} / \mathrm{cm}^{3}\right)$ and we investigated the film microstructure with AFM. Figure 4 shows the typical topography and magnetic domain structure of our films imaged in the demagnetized (as-prepared) state by AFM/ MFM, using the tapping-lift mode. The morphology of the scanned area exhibits a single crystal structure with an extreme smooth surface. The surface roughness was estimated to be as low as $0.23 \mathrm{~nm}$. The structure of our films illustrates a two-dimensional character, where the shape anisotropy is estimated to be $-2 \pi M^{2}=-0.64 \times 10^{5} \mathrm{~J} / \mathrm{m}^{3}$. On the other hand, the magnetic image [Fig. 4(b)] reveals magnetic domains with a clusterlike structure. For thinner films, the magnetization orientation in each cluster is confined up or down due to the strong perpendicular anisotropy. However, in thicker films, the clusterlike structure persists but the contrast of the magnetic image is less pronounced due to the weakness of the out-of-plane component of anisotropy. No stripe structure has been observed in our films, even in those with a strong perpendicular anisotropy. It is important to mention that stripe structure exists in films with a low coercivity and a pronounced shoulder at the nucleation field, ${ }^{7}$ which is unlikely to be the case in our samples (see Fig. 1). The impor- 


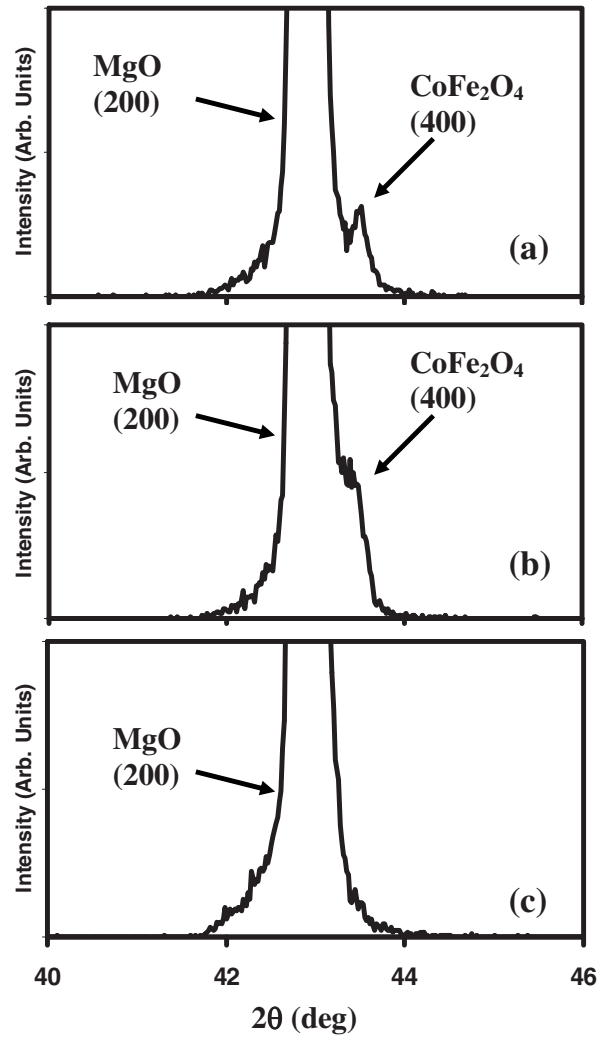

FIG. 5. $\theta / 2 \theta$ scan of (a) $60 \mathrm{~nm}$, (b) $240 \mathrm{~nm}$, and (c) $400 \mathrm{~nm}$ $\mathrm{CoFe}_{2} \mathrm{O}_{4}$ thick films. The shift of the film peak upon increasing the film thickness reveals a progressive change in the lattice dimension. Note that the lattice parameter normal to the film plane $\left(a_{\perp}\right)$ cannot be determined for the $400 \mathrm{~nm}$ thick film due to the overlapping between the film and the substrate peaks.

tance of $K_{v}$ is strongly dependent on the lattice state within the material. In a stress-free material, $K_{v}$ can be identified as the magnetocrystalline anisotropy, which is cubic in our case. However, in low dimensional systems such as thin films, the lattice strain can generate an additional source of anisotropy depending on the amplitude of the magnetoelastic effects. XRD has been used to estimate the strain magnitude in our layers. Figure 5 shows the XRD spectra $(\theta / 2 \theta$ scan $)$ of films having three different thicknesses $(60$, 240, and $400 \mathrm{~nm}$ ). It is important to notice the following effects in all the spectra regardless the film thickness: (a) $\mathrm{CoFe}_{2} \mathrm{O}_{4}$ constitutes the unique phase in our layers and (b) all the manifested Bragg reflections are parallel to the (100) texture. The very narrow rocking curve $\left(0.16^{\circ}\right.$ as full width at half maximum) confirms a highly oriented structure of the films. The high quality of epitaxy is confirmed by the inplane measurement, where the $\varphi$ scan of the (511) texture reveals a fourfold symmetry with a perfect alignment between the film and the substrate peaks. However, Fig. 5 shows a significant dependence of the $\mathrm{CoFe}_{2} \mathrm{O}_{4}$ peak position on the film thickness. For the thinner layer $(60 \mathrm{~nm})$, the (400) peak of cobalt ferrite appears to be well separated from the substrate peak, whereas upon increasing the film thickness, it is significantly shifted to become a shoulder of the (200) $\mathrm{MgO}$ reflection [see Fig. 5(b)]. At large thickness $(400 \mathrm{~nm})$ [Fig. 5(c)], the shift of the film peak is even more

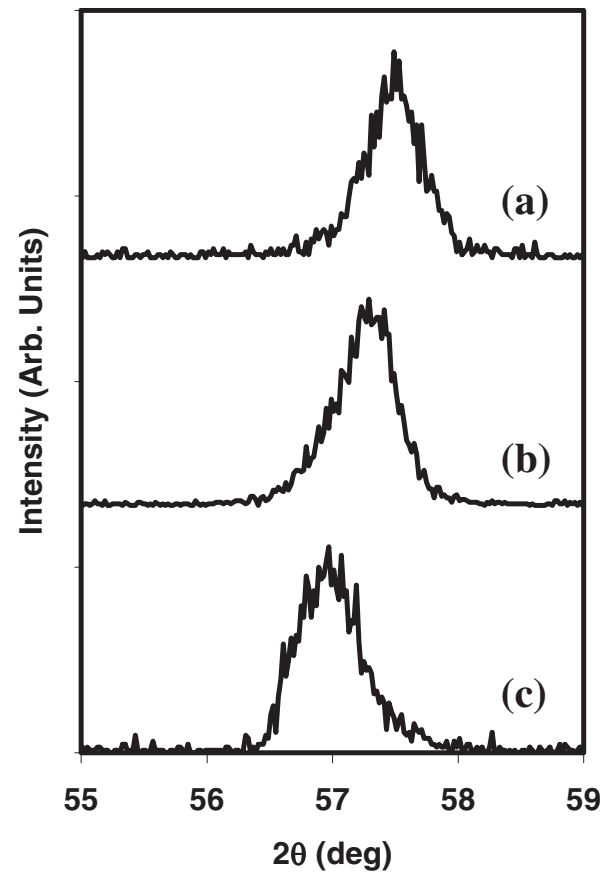

FIG. 6. Asymmetric scan of the (511) texture in (a) $60 \mathrm{~nm}$, (b) $240 \mathrm{~nm}$, and (c) $400 \mathrm{~nm} \mathrm{CoFe} \mathrm{O}_{4}$ thick films. The shift of the (511) peak is indicative of a continuous transition from a distorted lattice at small thickness toward a relaxed cubic lattice at large thickness.

pronounced and both reflections (film and substrate) overlap. The significant shift of the (400) peak in Fig. 5 illustrates an important lattice relaxation in the film. For example, changing the film thickness from 60 to $240 \mathrm{~nm}$ induces an increase in the lattice parameter normal to the film plane $\left(a_{\perp}\right)$ from 8.339 to $8.358 \AA$. Since the only planes reflecting in the $\theta / 2 \theta$ scan configuration are those parallel to the film plane, additional measurements are required to estimate the lattice parameter parallel to the film plane $\left(a_{\|}\right)$and to establish whether stress is tension or compression. Figure 6 shows the thickness dependence of the (511) $\mathrm{CoFe}_{2} \mathrm{O}_{4}$ peak in the asymmetric scan configuration. The shift of the (511) peak is a good illustration of the lattice relaxation upon increasing the film thickness. By combining both scans (symmetric and asymmetric), $a_{\|}$was determined for 60 and $240 \mathrm{~nm}$ thick films. However, it was impossible to evaluate the lattice parameters for the $400 \mathrm{~nm}$ thick films from both XRD scans due to the overlapping between the film and the substrate peaks in the symmetric scan. By considering the relation between both strains $\left[\varepsilon_{1}=\left(a_{\|}-a_{0}\right) / a_{0}\right.$ and $\varepsilon_{2}=\left(a_{\perp}-a_{0}\right) / a_{0}$ are the in-plane and the out-plane strains of the film, respectively, in regard to the unstrained lattice $a_{0}$ of the bulk] and the Poisson ratio $\nu$ (estimated for other thinner films to be 0.333 ), we were able to establish a second equation $\left[\varepsilon_{1} / \varepsilon_{2}=2 \nu /(\nu-1)\right]$ for the $400 \mathrm{~nm}$ thick film and determine the lattice dimensions at that thickness. The summary of the lattice size is given in Table I, which reveals the following effects. (a) The difference between $a_{\perp}$ and $a_{\|}$confirms a tetragonal distortion of the film lattice, which is strongly dependent on the film thickness. (b) The large value of $a_{\|}$in the thinner film (8.42 $\AA$ ) in comparison with the bulk value $(8.38 \AA)$ (Ref. 8$)$ is a clear indication that the in-plane 
TABLE I. Thickness dependence of the lattice dimensions and the corresponding magnetoelastic anisotropy estimated from calculation. The strain anisotropy is large enough to overcome the shape anisotropy in a thinner film, whereas it is largely diminished to be dominated by the shape anisotropy in a thicker film.

\begin{tabular}{lccccccc}
\hline \hline $\begin{array}{l}\text { Thickness } \\
(\mathrm{nm})\end{array}$ & $\begin{array}{c}a_{\|} \\
(\AA)\end{array}$ & $\begin{array}{c}a_{\perp} \\
(\AA)\end{array}$ & $\begin{array}{c}\left(a_{\|}-a_{\perp}\right) \\
(\AA)\end{array}$ & $\begin{array}{c}\text { In-plane } \\
\text { strain } \\
(\%)\end{array}$ & $\begin{array}{c}\text { Out-of-plane } \\
\text { strain } \\
(\%)\end{array}$ & $\begin{array}{c}\text { Strain } \\
\text { anisotropy } \\
\left(\mathrm{J} / \mathrm{m}^{3}\right)\end{array}$ & $\begin{array}{c}\text { Shape } \\
\text { anisotropy } \\
\left(\mathrm{J} / \mathrm{m}^{3}\right)\end{array}$ \\
\hline 60 & 8.42 & 8.339 & 0.081 & 0.477 & -0.489 & $14.0 \times 10^{5}$ & $-0.64 \times 10^{5}$ \\
240 & 8.401 & 8.358 & 0.043 & 0.250 & -0.262 & $7.41 \times 10^{5}$ & $-0.64 \times 10^{5}$ \\
400 & 8.381 & 8.379 & 0.002 & 0.012 & -0.012 & $0.35 \times 10^{5}$ & $-0.64 \times 10^{5}$ \\
Bulk & 8.380 & 8.380 & 0 & & & & $-1 \times 10^{5}$ \\
\hline \hline
\end{tabular}

stress is tension rather than compression. The tetragonal distortion of the lattice suggests that the magnetoelastic energy could be actively involved in the control of the spin alignment. According to Schulz and Baberschke ${ }^{9}$ and Thamankar et al. ${ }^{10}$ the magnetoelastic anisotropy energy associated with the tetragonal distortion of the lattice in a (100) oriented film is formulated as $K_{\mathrm{me}}=3 / 2\left[\lambda_{[100]}\left(C_{11}-C_{12}\right)\left(\varepsilon_{2}-\varepsilon_{1}\right)\right] . \lambda_{[100]}$ is the magnetostriction constant along the [100] crystallographic direction, and $C_{11}$ and $C_{12}$ are the elastic constants. From the expressions of both strains $\left(\varepsilon_{1}\right.$ and $\left.\varepsilon_{2}\right)$, the magnetoelastic anisotropy can be directly related to $\left(a_{\|}-a_{\perp}\right)$, as expressed by the following formula: $K_{\text {me }}=-3 / 2 \lambda_{[100]}\left(C_{11}-C_{12}\right)\left(a_{\|}-a_{\perp}\right) / a . K_{\text {me }}$ was calculated in our case by assuming the bulk values of cobalt ferrite $\left(C_{11}=2.7 \times 10^{12} \mathrm{dyn} / \mathrm{cm}^{2}, \quad C_{12}=1.06 \times 10^{12} \mathrm{dyn} / \mathrm{cm}^{2}\right.$, and $\left.\lambda_{[100]}=-5.9 \times 10^{-4}\right) .{ }^{11}$ Since $\lambda_{[100]}$ is negative, two different spin configurations can be stabilized in our (100) $\mathrm{CoFe}_{2} \mathrm{O}_{4}$ films. (1) If the in-plane stress (along the [010] and [001] directions $)$ is tension, $\left(a_{\|}-a_{\perp}\right)$ is positive, leading to positive $K_{\text {me }}$. Consequently, the generated magnetoelastic anisotropy will be uniaxial and oriented perpendicular to the film plane. (2) If the in-plane stress is compression, $\left(a_{\|}-a_{\perp}\right)$ is negative $\left(K_{\mathrm{me}}<0\right)$, leading to a biaxial in-plane anisotropy. The first scenario is more likely to match our case, where the in-plane stress is tension rather than compression. As reported in Table I, the estimated values for strain anisotropy seem to comfort the out-of-plane torque measurement. In fact, the existence of a large rotational hysteresis in the torque curve results from a nonsaturation regime, where the intrinsic anisotropy field $H_{k}$ is much larger than the applied field of the measurement. As illustrated in Table I, the magnetoelastic energy is strong enough to overcome the shape anisotropy at small thicknesses. However, the drastic reduction in the strain anisotropy at large thicknesses allows the shape anisotropy to be more competitive, leading to an in-plane align- ment of the magnetization. The large magnetoelastic effects manifested in our structures could be explained from the growth mechanism. As reported earlier, the small lattice mismatch between $\mathrm{CoFe}_{2} \mathrm{O}_{4}$ and the substrate may favor a pseudomorphic growth, where the in-plane film lattice adopts the size of four lattice units of $\mathrm{MgO}$ at small thickness. Consequently, the film lattice will experience a tensile stress, which generates a perpendicular anisotropy as predicted before. Upon increasing the film thickness, the lattice strain will be progressively released via dislocations, leading to a cubic structure, where the magnetization will be forced by the shape anisotropy to align along the film plane. In fact, the coexistence of two competing uniaxial anisotropies as illustrated by the out-of-plane torque curves is a clear indication of the coexistence of two phases (tetragonal and cubic) in our layers. The tetragonal phase dominates at small thicknesses, whereas the cubic regime (relaxed lattice) prevails at large thicknesses. As reported in Table I, the highest anisotropy generated by strain $\left(1.4 \times 10^{6} \mathrm{~J} / \mathrm{m}^{3}\right)$ approaches the magnitude of the uniaxial anisotropy of FePt stabilized in the $L 1_{0}$ tetragonal phase $\left(4 \times 10^{6} \mathrm{~J} / \mathrm{m}^{3}\right){ }^{12}$ The superparamgnetic limit in our highly strained film is expected to be as small as $4.1 \mathrm{~nm}$, which is still larger than that typically known for the FePt phase $(2.9 \mathrm{~nm})$.

In conclusion, spin reorientation has been reported in epitaxial $\mathrm{CoFe}_{2} \mathrm{O}_{4}$ thin films upon varying the film thickness. The driving force of such a phenomenon is established to be the lattice strain. The tetragonal distortion of the lattice at small thickness generates a perpendicular anisotropy, large enough to overcome the shape anisotropy and to stabilize the magnetization out of plane. However, in thicker films, the lattice relaxation toward the cubic structure of the bulk allows the shape anisotropy to force the magnetization to be in plane aligned.
*FAX: (443) 885-8288; alisfi@ morgan.edu

${ }^{1}$ J. Pommier, P. Meyer, G. Penissard, J. Ferre, P. Bruno, and D. Renard, Phys. Rev. Lett. 65, 2054 (1990); R. Allenspach, M. Stampanoni, and A. Bischof, ibid. 65, 3344 (1990).

${ }^{2}$ M. Speckmann, H. P. Oepen, and H. Ibach, Phys. Rev. Lett. 75, 2035 (1995).
${ }^{3}$ A. Lisfi, J. C. Lodder, H. Wormeester, and B. Poelsema, Phys. Rev. B 66, 174420 (2002).

${ }^{4}$ M. J. Carey, S. Maat, P. Rice, R. F. C. Farrow, R. F. Marks, A. Kellock, P. Nguyen, and B. A. Gurney, Appl. Phys. Lett. 81, 1044 (2002); S. Maat, M. J. Carey, E. E. Fullerton, T. X. Le, P. M. Rice, and B. A. Gurney, ibid. 81, 520 (2002). 
${ }^{5}$ A. Lisfi, J. C. Lodder, E. G. Keim, and C. M. Williams, Appl. Phys. Lett. 82, 76 (2003); A. Lisfi and J. C. Lodder, J. Phys.: Condens. Matter 14, 12339 (2002).

${ }^{6}$ E. C. Stoner and E. P. Wohlfarth, Philos. Trans. R. Soc. London, Ser. A 240, 599 (1948).

${ }^{7}$ C. Kooy and U. Enz, Philips Res. Rep. 15, 7 (1960); M. Hehn, S. Padovani, K. Ounadjela, and J. P. Bucher, Phys. Rev. B 54, 3428 (1996); V. Gehanno, A. Marty, B. Gilles, and Y. Samson, ibid. 55, 12552 (1997).

${ }^{8}$ A. Goldman, Modern Ferrite Technology (Van Nostrand, New
York, 1990), p. 26.

${ }^{9}$ B. Schulz and K. Baberschke, Phys. Rev. B 50, 13467 (1994).

${ }^{10}$ R. Thamankar, A. Ostroukhova, and F. O. Schumann, Phys. Rev. B 66, 134414 (2002).

${ }^{11}$ M. D. Sturge, E. M. Gyorgy, R. C. LeCraw, and J. P. Remeika, Phys. Rev. 180, 413 (1969); Y. Suzuki, G. Hu, R. B. van Dover, and R. J. Cava, J. Magn. Magn. Mater. 191, 1 (1999).

${ }^{12}$ J. U. Thiele, L. Folks, M. F. Toney, and D. K. Weller, J. Appl. Phys. 84, 5686 (1998). 